the early part of decompression, and much too fast towards the end. To decompress safely a man after a long exposure at, e.g., $75 \mathrm{lb}$. pressure would probably require eight or ten hours if the pressure were reduced at a uniform rate throughout; by the new method here described not much more than three or four hours would be required. At such pressures it is clear that in practice the time of exposure must be reduced.

\section{THE DIET OF THE HINDU.'}

CHITTENDEN'S well-known work on diet advocates a reduction of the nitrogen intake to an amount far below that in the standard Voit dietary. By experiments on others and himself, he endeavoured to show that a low protein diet is compatible with bodily equilibrium and health. $\mathrm{He}$ directs attention to the well-known fact that the muscular energy of the body makes little or no call on the nitrogenous constituents of the muscles, and urges that the excretory channels, such as the kidneys, are overworked and so liable to damage when dealing with the large amount of waste nitrogren which it is the habit of the white races to ingest.

The conditions which Chittenden artificially constructed for the subjects of his experiments are found ready made on a large scale in the teeming millions of Bengal. It is quite obvious that much more correct conclusions as to the ultimate outcome of a reduction of the protein intake to less than half the European standard can be drawn from such a huge experiment, extending over the lives of a population, than it is possible to deduce from a limited series of observations on a few men lasting only for a few months. Scientific investigators therefore owe a debt of gratitude to Prof. McCay for the brilliant piece of work in which he has systematically and statistically grappled with the problem.

$\mathrm{He}$ admits it is perfectly true that the Bengali of varying castes maintains nitrogenous equilibrium on his poor vegetarian diet, and he naturally does not dispute the dictum that nitrogen is not the source of muscular energy. But his conclusion is that the low nitrogen intake acts deleteriously: the amount of protein in the blood is permanently decreased in amount, and the poor pabulum provided for the muscles leads to work in disadvantageous circumstances, and tends to produce degenerative changes in the body cells, notably in the kidneys. Not only is the output of work by the Hindu miserably small in comparison with the European labourer, but he is more easily fatigued, his blood-pressure is below the normal, and his lack of reserve force renders him an easy prey to bacterial infection and other forms of disease. His body weight and measurements show the native to be a puny person, exhibiting all those signs usually associated with an under-fed condition.

1 Scientific Memoirs by the Officers of the Medical and Sanitary Defartments of the Government of India (No. 34). Standards of the Constituents of the Urine and Blood and the bearing of the Metabolism of Bengalis on the Problems of Nutrition. By Capt. D. McCay. Pp iv $+67 . \quad$ (Calcutta Superintendent Government Printing, India, 1908.) Price $\frac{1}{4} s .2 d$.
Even if one grants that in the normal decomposition of proteins, toxic substances are formed which may act injuriously upon the body, it must be remembered that in health the body is provided with an efficient machinery for eliminating them. It is by no means certain that decomposition products, also toxic in nature, are not formed from the fat and carbohydrate of the diet. The large carbohydrate intake rendered necessary by a diet poor in nitrogen seems to be full of danger, and the extreme prevalence of diabetes in its most aggravated forms among the Bengalis proves conclusively that the evils due to excess of carbohydrate are even more real than those supposed to be due to excess of protein.

The favourite argument of the vegetarian is to point to the races of the east as examples of the good results accomplished on a low diet. Such an argument has been refuted more than once, and its fallacies have been finally demonstrated by Prof. McCay's observaticns and statistics.

W. D. H.

\section{THE SPECTRA OF THE MAJOR PLANETS.}

$A \mathrm{~S}$ the result of his experiments, Mr. V. M. Slipher A succeeded in 1907 in rendering some plates (Seed, 23) sensitive far into the red. This was done by bathing them before exposure in a solution of pinacyanol, pinaverdol, dicyanin, alcohol, and water. With them he photographed the spectra of all the major planets and, for comparison purposes, that of our moon.
The results are shown in the accompanying print, made by enlarging, combining, and re-photographing on one plate all the spectra. Three things are to be observed in the spectra :-

(i) The great number of new lines and bands disclosed. Some of these are evident in the spectra of Jupiter and Saturn, but chiefly in those of Uranus and Neptune.

(2) A steady progression in the intensities of the non-solar lines and bands as one goes outward from the sun.

(3) The intensification of the hydrogen lines $\mathrm{F}$ and $\mathrm{C}$, notably in Uranus and Neptune.

\section{Percival Lowell.}

